\begin{tabular}{|c|c|c|}
\hline & Mansoura University \\
Faculty of Engineering & Mansoura Engineering Journal & 0 \\
\hline
\end{tabular}

\title{
Optimizing Public Transportation Networks Using Space Syntax as an Evidence-Based Approach: The Case Study of Mansoura City
}

\author{
Sara Elgamal, Shrief Sheta, and Mohanad Fouda
}

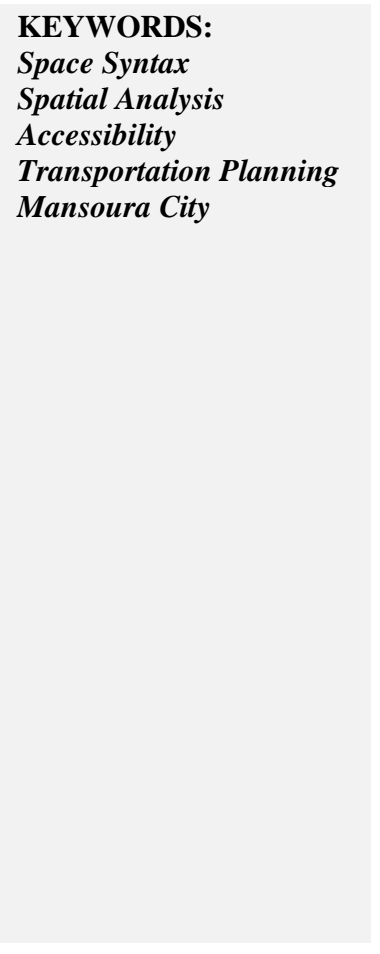

\begin{abstract}
Transport network planning in Egypt suffers from insufficient planning that should be done prior to execution. This research presses on the fact that personal experience and cognition alone cannot be the main factors of design that it should go hand in hand with scientific measurable analysis and evidence. Hence, it focuses on having a deeper understanding of the influence of spatial network configuration on urban activity and centrality. It proposes that Space Syntax, as a syntactic model, has potential to be utilized in optimizing transportation networks, based on Space Syntax ability to model and predict streets with most urban activity and areas that act as central gathering points, and therefore, giving a scientific basis to evident decisions to choose optimum routes and stations for transit networks. The study hypothesizes that adding Space Syntax spatial configuration analysis as a layer to the whole process can give a better understanding of the network. This research applies Space Syntax configurational analysis with the aim to present an evidence approach to optimize its transportation network. The proposed approach is applied on Mansoura City, an intermediate city in Dakahlia governorate in Egypt. The methodology includes field observations using gate counting technique to identify movement flows in different parts of the city, running a segment map analysis of the city's street network and validating the results of the analysis through comparing computed values with observations. Results identify streets with major urban movement which represent potential transit routes and areas that are considered urban centers which represent potential transit stations. These results verify the important role of accessibility analysis in transportation planning, and therefore presents an applicable approach to identify optimum transit routes and stations that have higher potential to act as active movement corridors at a city scale.
\end{abstract}

Received: (7 June, 2020) - Revised: (7 October, 2020) - Accepted: (9 October, 2020)

Corresponding Author: Sara Elgamal is a Teaching Assistant at the Architecture Department, Faculty of Engineering at Mansoura University, Mansoura,Egypt (e-mail: sara_elgamal@mans.edu.eg)
Shrief Sheta is an Associate Professor with the Architecture Department, Faculty of Engineering at Mansoura University, Mansoura, Egypt (e-mail: Sheriefsheta@mans.edu.eg)

Mohanad Fouda is a Lecturer with the Architecture Department, Faculty of Engineering at Mansoura University, Mansoura, Egypt (e-mail: Mohanadfoda@mans.edu.eg). 


\section{INTRODUCTION}

$\mathrm{T}$ HE Inappropriate planning of transportation networks in Egyptian cities has led people to resort more to private cars which as a result caused many negative consequences, such as congestion, increased rate of accidents, and increased rate of harmful emissions. In order to shift the dependency away from private vehicles, cities must invest in sustainable mobility [1]. In order to achieve sustainable mobility, planners and policy makers have exhorted efforts in planning efficient infrastructures with the aim of reducing emissions related to transport and shifting to public modes of transport [2].

Until now, traditional transit planning mostly applies caroriented planning while envisioning infrastructure for transit [3], where priority is given to private vehicles rather than public transportation modes. This method creates difficulties later on, when aligning routes and stations with the planned network. Recent studies focused on the challenges in conventional transport planning models owing to their complexity and the amount of time it needs to run [4]. Researchers have suggested models that are simpler that provide an easier way for decision makers to perceive urban networks. Research in urban spatial morphology has highly advanced measures which enable researchers and planners to quickly better understand potentials of accessibility in different areas of cities and spatial structures [5]. These advanced measures can be put to good use in transit planning. This research focuses on the potential of using spatial configuration analysis developed by Space Syntax as an approach to identify and evaluate optimized routes and stations for public transportation networks. The case study examined in this research is Mansoura City, an intermediary city in Dakahlia governorate in Egypt. The main objective of applying Space Syntax measures on the spatial structure of the city is to identify the optimum routes and stations based on two pivotal indicators which are urban activity and centrality.

The predominant question this research attempts to answer is what are the potentials and limitations of Space Syntax as an approach in the process of optimizing public transportation networks? The study answers this by discussing wider characteristics of planning with a focus on the ability to identify corridors of urban activity and areas of urban centers using Space Syntax indices. The paper is structured in the following way: the first section lays out the theoretical basis for the research. The following section explains the used methodology, observation techniques used and the analysis conducted on the previously mentioned case study. Following that, the results of the analysis are elaborated, and validated by comparing the computed analysis results and observations. The validation step is very important to prove the accuracy of Space Syntax spatial analysis in modeling movement patterns, therefore denoting its potential use in transit network planning. The research ends with a discussion and conclusion of the potentials and limitations of the proposed approach and its applications.

\section{TheOretical BACKGROUND}

In this section, the research gives a brief overview of the conventional transportation planning models while mentioning the aspects that distinguish syntactic models from traditional ones. Then, Space Syntax is further demonstrated as one of the most advanced syntactic models that have had many applications in different urban planning aspects including transport network planning. The aim of this section is to build a theoretical foundation for the study.

\section{A. Traditional transportation network planning approach}

There are three main pillars that are vital for transit planning which are: forecasting trip demand, service operation and urban policies [6]. Forecasting demand concerns evaluating demand using Transportation/land-use modeling (TLUM). On the other hand, service operation concerns feasibility and setting alternatives in early planning phases, while urban policies confronts the different urban and transport objectives of each transit alternative [7]. Following this framework, the first step is mapping potential routes from the perspective of physical feasibility. The second is proposing different alternatives of routes and stations using a multi-criteria evaluation that measures various factors such as: catchment area of stations, costs of construction of routes and stations, and network configuration features as connectivity and directness. Then comes the role of the team of planners to decide, using a multi criteria analysis, on a preferred alignment based on the urban policies objectives [8].

However, travel demand is not the only aspect served by transport infrastructure, since it also forms the city and influences various urban phenomena [9]. TLUM has a major flaw in practice which is that it doesn't take into consideration land use potential that may occur due to changes in infrastructure. Current models of transport rely on land-use and demographics in a one-way direction. Which means TLUM neglects areas that has inherent potential for urban densification. In addition, the element of accessibility is only assessed from a limited view. Therefore, speaking in terms of accessibility, it's doubtful that TLUM can generate the optimum network.

This literature overview highlights the point that distinguishes syntactic models, such as Space Syntax and others, from conventional transport models. While conventional models focus on demographics, syntactic models focus of topological analysis of spatial networks in order to measure accessibility. The main concept of syntactic research has been claimed by Lerman and Omer that urban configuration can be the best predictor of movement and flow and combining quantitative properties of the configuration with demographics and land-use can make the predictions more accurate [10].

\section{B. Space Syntax and Urban Movement}

Space Syntax is a science-based and human focused approach to analyzing spatial configurations. It is built on a set of theories correlating society with space and a set of techniques and tools for analyzing spatial structures. Space Syntax focuses on describing how the configuration of space directly impacts how people understand, use and move through spatial settings of all kinds, ranging from small-scale spaces to large-scale 
urban cities [5]. Space Syntax is well developed as a spatial configuration approach, which relies mainly on analyzing the topological characteristics of a street network and explain most of the spatial movement in the urban structure, including both vehicles and pedestrians, based on these characteristics [11]. It is not only movement that is affected by the street network configuration but also the distribution on land uses and other spatial phenomena [12].

Spatial configuration analysis of Space Syntax was based on the theory of "natural movement". This theory is based on the main proposition that there is a correlation between grid configuration and pedestrian movement patterns [13]. Built on that, the theory of "cities as movement economy" proposes that the configurational structure of space is a main generator of different patterns of movement. As a result, influencing land use choices. In turn, creating a multiplier effect on movement with further influence of land use choices [14]. Configurational analysis has been used as an approach to predict pedestrian movement in different areas. In London, various studies have been carried out to predict pedestrian movement with predictably rates ranging from 55-75\%. Meanwhile, in Amsterdam the rates of predictability of pedestrian movement ranged from $60-70 \%$ [11].

\section{- Predicting Urban Activity}

The analysis of Space Syntax demonstrates the relative accessibility of different areas of the urban configuration through quantifying and measuring various characters of the urban network. An axial map analysis illustrates the level of visual and movement accessibility from the moving user's perspective. The construction of an axial map is basically a representation of the street network by the fewest and longest straight lines that completely covers all open spaces of the urban network, entitled axial lines. When the topological relationship between these axial lines are analyzed several indices are generated describing the street network. These indices include: connectivity, integration, choice and others. Each index reflects a specific and measures a certain aspect [15].

Space Syntax research stresses on the idea that movement and flow in urban structures are largely formed by its spatial configuration. Various analytical tools were developed based on the theory of Space Syntax for analyzing urban configuration in order to understand the correlation between the city's form and its function. Which gave the theory a wide range of applicability in urban planning and design. The results of these configurational analysis are represented in numerical values and maps with a wide color range representing various indices. Where routes which are more accessible and connected are red and routes that are segregated and less accessible are blue [16].

\section{Space Syntax and Centrality}

The theory of centrality as a process proposes that urban centers are formed and located as an output of a continuing historical process. The process involves the movement patterns which are shaped initially by the spatial configuration, which subsequently impact land use distribution in the network from busier to quieter areas. As a result, influencing land use choices and generating areas that act as attractors in the whole settlement area. The process responds to configurational properties of the spatial layout and at the same time introduces changes in is by adjusting the local grid conditions. Typically, the street network is locally intensified in some areas as a form of adaptation through creating small scale urban blocks, which are more permeable and accessible [17].

\section{- Predicting Centers and Sub-Centers}

Space Syntax has developed unique methods to analyze spatial structures and identify centrality. The methods include a group of graph-based analytical features. These features include that the street segment is considered as an urban unit itself instead of the connection points of the streets and secondly the way it measures distance is by steps or angular changes instead of metrics. Detecting the main spatial distribution of pedestrian activity is very useful and essential in transportation network planning. Angular closeness centrality, which is the measure used in this study to identify centers and sub centers, measures the potential of each street segment, owing to angular proximity, to all other segments within a specified radius [18].

\section{Case Study in Mansoura City}

\section{A. Background}

The study is conducted on Mansoura city, the capital of Dakahlia governorate. It is considered as an intermediary city in Egypt, with an estimated population in 2018 of more than half a million [19]. Mansoura is located in the Delta region (see fig.1), on the east bank of Damietta branch. It's nearly $120 \mathrm{~km}$ northeast of Cairo. On the other side of the Nile, is located a town named Talkha which is connected to Mansoura by bridges across the Nile river. Mansoura city has an important provincial role as it is the administrative center of Dakahlia governorate. The city is also the center of medical, commercial, educational and administrative services for its residents and all the surrounding sub-urban areas and villages [20].

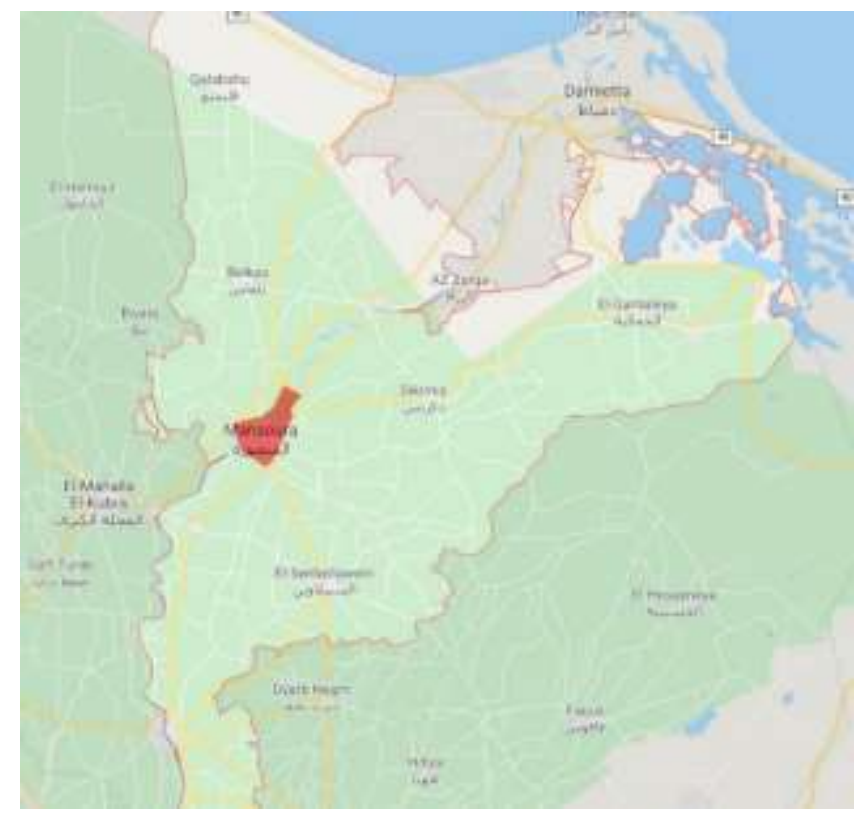

Fig. 1. Mansoura City Location in the Dakahlia Governorate Source: Google maps (www.google.com/maps/). Accessed: April 2020 
Mansoura has a wide range transit modes which can be categorized into inner-city modes such as public buses, microbuses and a newly established Nile river transit, and transit modes connecting the city with surrounding rural areas, cities and other governorates such as railway trains and buses.

Generally speaking, in Egypt, the process of planning transit routes starts by assessing the needs of a given neighborhood. First, the local administrative unit concerned with the matter files a request which is then assessed by a technical staff from the traffic administration to investigate and gather data about the population size and economic conditions of those who live in the neighborhood. Based on the collected data, if the population size is large, the traffic administration will operate routes in the given neighborhood. The transit routes and the allocation of transit stations are determined by the traffic administration. Even though, it may seem like the process is evidence-based, there is no clear criteria and the assessment appears to be superficial [21]. This study aims at proposing an evidence based approach to planning transportation networks through spatially analyzing the road network of Mansoura city in order to identify roads that have good potential for transit routes and areas that have good potential to have transit stations based on Space Syntax spatial measures.

\section{B. Methodology}

In order to start the spatial analysis of the given case study in Mansoura city, the study starts by collecting spatial data such as maps and main street network. A significant part of data collection is field observations using gate counts technique done by the researchers in order to manually measure movement flow pattern in different areas across the city, these measures will later be used to validate the computed values. After data collection, the network is spatially represented in the form of an axial map based on the theories and tools provided by Space Syntax. Then the spatial analysis is conducted on both global and local scales, in order to identify both urban activity corridors and urban activity centers. Results of global scale spatial analysis assists in choosing potential transit routes, while local scale spatial analysis assists in determining potential transit stations. Visualizations of these results can be utilized in the decision-making process, inform policy makers and enhance stakeholder's participation.

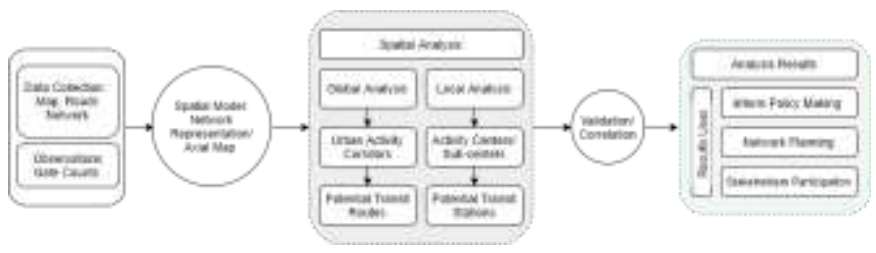

Fig. 2. Proposed Methodology for Applying the Syntactic Model in Optimizing Transportation Networks Source: The Authors

\section{Observations}

The observation technique used in this study is "Gate Counts". It is a technique developed by University College London (UCL) Space Syntax Laboratory that is used to observe pedestrian and vehicular movement patterns and flow in urban layouts and buildings. A gate is defined as an imaginary line, drawn by the observer, which crosses the street or space. In order to identify movement volume, at this gate the observer counts how many pedestrians or vehicles cross. To conduct a thorough observation, a number of gates that cover the whole study area must be selected. The selected gates must represent a variety of spaces that range in activity [22].

Observations were done on weekdays during peak hours, between 1:00 pm and 3:00 pm, 20 gates were selected in different areas of the city that have different levels of pedestrian and vehicular flow, a sample of the chosen gates are illustrated in table (1). It is important to note that these observations were done in April 2020 during the COVID-19 outbreak so they do not represent the usual numbers on ordinary days, however they do represent the relative flow of the city's network, see fig.(3) which is based on the observed flow noted in table (1). We were not able to make observations on the weekends due to the necessary lockdown precautions taken by the government to avoid the spread of the virus.

TABLE I

Gate Counts Sample Measuring: Pedestrian Flow PeR hour (P) \& VEHICULAR FlOW PER HOUR (V). SOURCE: THE AUTHORS

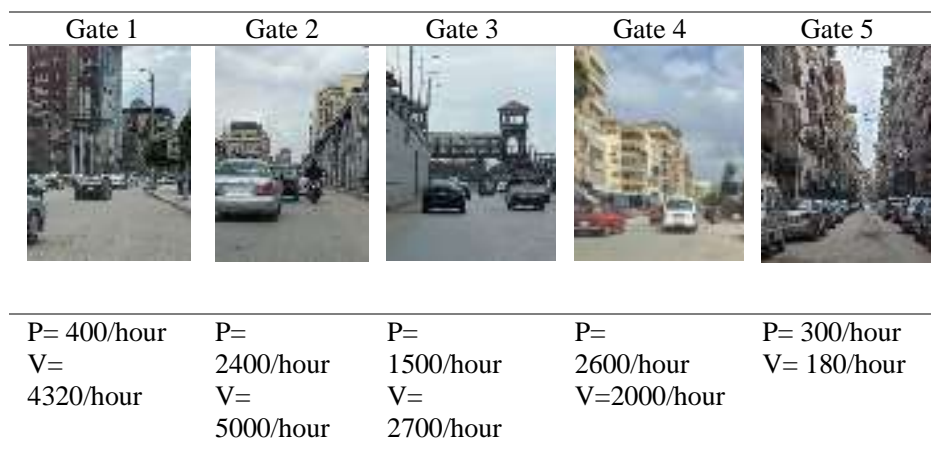

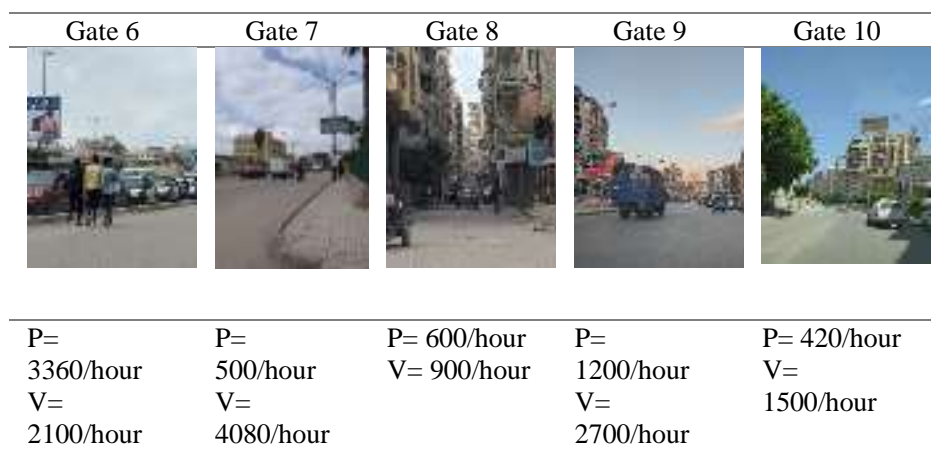




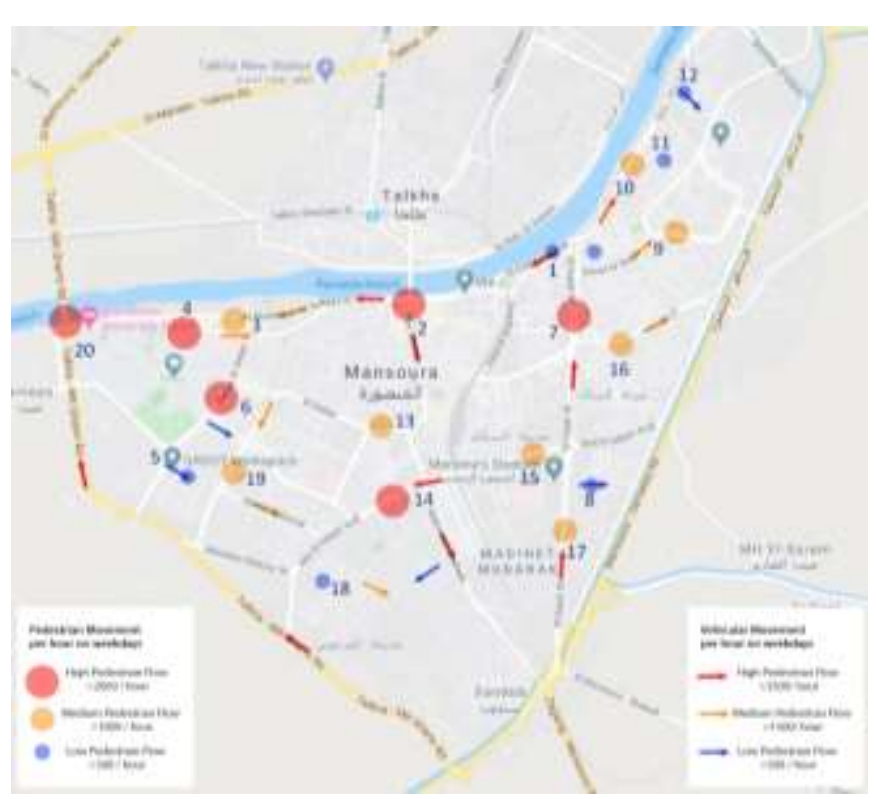

Fig. 3. A map describing average pedestrian volume on weekdays. Base map: Google Maps (www.google.com/maps/). Accessed: April 2020. Source: The Authors

\section{Analysis}

\section{1) Creating axial map}

The first step starts by manually generating an axial map of the city based on the road centerline network we gained from Open Street Maps, see fig. (4) \& (5). The second step was converting the axial map to a segment map in DepthmapX software. DepthmapX is a software developed by UCL based on the theory of Space Syntax for spatial network analysis. The measures are represented numerically and by a color range ranging from red to blue which represent values from high to low respectively [22]. (see fig.6)

\section{2) Segment map analysis}

The segment map is originated from the axial map. While axial maps are considered the representation of the fewest number of main routes inside the urban network, segments map is considered as a more detailed representation where lines are fragmented into several segments depending on the nodes that exist in the grid. Therefore, using segment maps correlates Space Syntax representation of space with those used in traffic planning, which is mainly built on links as segments and nodes as connections. The calculations of a segment map is based on measuring mean depth degree of the road network. For example, deeper routes which are more segregated tend to be used less, while integrated routes tend to be used more frequently [23].

Segment map analysis of Mansoura city was done using DepthmapX software. Based on the exported map from Open Street map (fig.4), an axial map was generated manually in order to be used in the analysis (fig.5). Segment map analysis was conducted to identify which part of the urban network is more integrated and possess more urban activity and movement. A color spectrum is used to present the degree of integration of streets. Where red is assigned to the most integrated streets, ranging down to blue which are assigned to streets that are less integrated into the network [24].

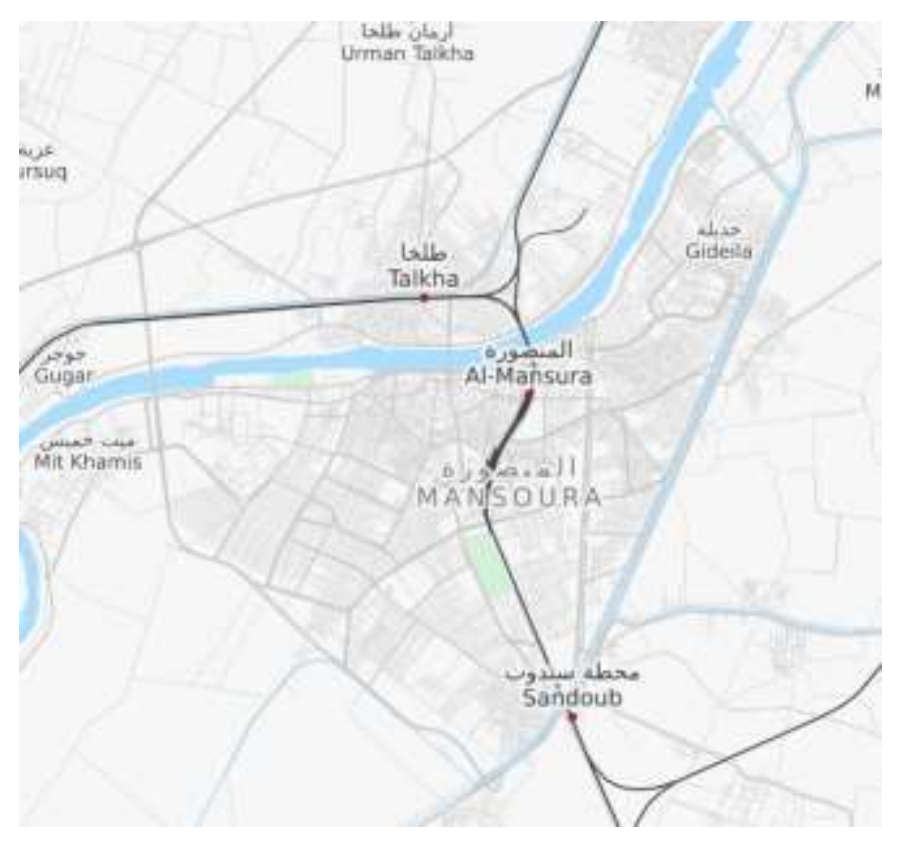

Fig. 4. Mansoura City Road Network. Source: Open Street Maps (www.openstreetmap.org). Accessed: April 2020.

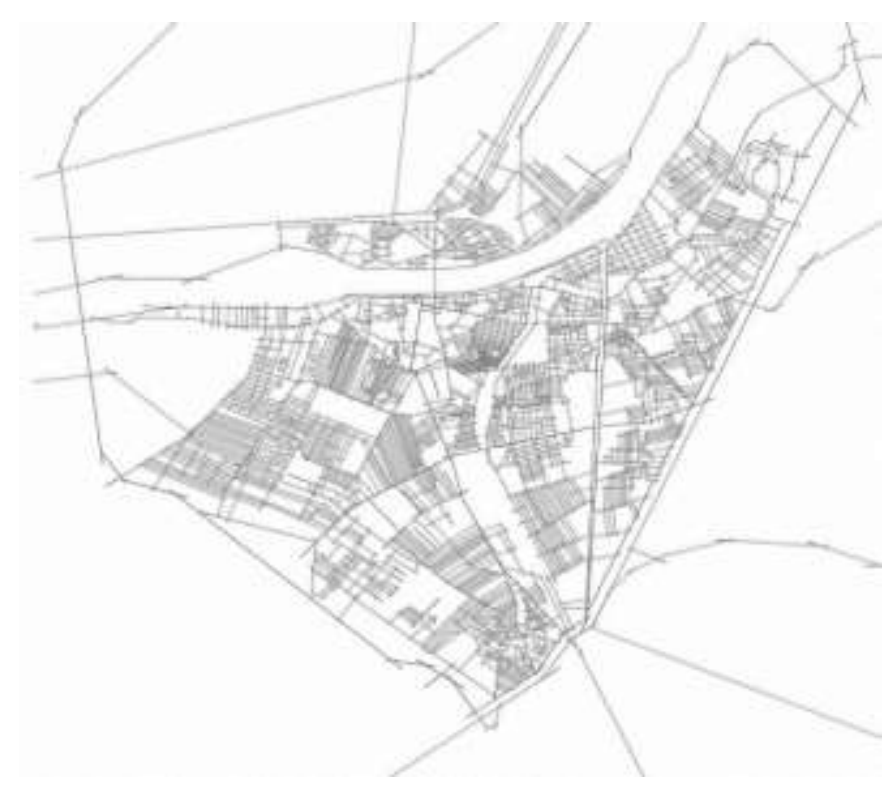

Fig. 5. Axial Map, Spatial representation of Mansoura city. Source: The Authors, using Auto Cad software overlaying the Open Street Map. Date: April 2020

\section{Spatial Analysis Results}

The analysis results of a syntactic model is highly dependent on the scale of analysis. Which means that the results will 
represent movement patterns in relation with the chosen scale. Ranging from walkable scales to regional vehicular travel patterns. When using syntactic model analysis is transportation network planning different scales can be utilized for different reasons. Short radii that range between $200 \mathrm{~m}-1000 \mathrm{~m}$, which is considered as a walkable distance, can identify local accessibility that can be considered ad distance to transit. On the other hand, large radii that are more than $2 \mathrm{~km}$ can be used to identify destination [25].

\section{A. Urban Activity Index and Optimized Routes}

The index of urban activity represents the potential volume of activity occurring on different routes of the urban area. Its calculation is done through normalizing, at respective radii, weighted choice values with values of total depth. Normalized angular choice (NACH) value are used in this study because it provides more clear and consistent results than raw values.

The equation (1) is used to calculate normalized choice at a global scale where $r=n$

NAChoice_n= log $\left(A C h \_n+1\right) / \log \left(A T D \_n+3\right)$

Where:

- The radius (n) is a representative of a large radius that takes the segments of the whole spatial structure into analysis.

- ACh_n: angular choice at radius =n

Angular Choice is a measure that represents how likely a segment will be passed through from all other spaces to reach any other space, taking into consideration the shortest path or least number on angular turns with in a given set radius [12].

- A TD_n: angular total depth at radius $=\mathrm{n}$

Angular Total depth is the sum measure of the shortest angular steps from any segment in the network to a selected segment [24].

The resulted measures of normalized choice is visualized in a map where routes are given a color range which varies from red to blue (see fig.6). The values of $\mathrm{NACH}$ range from 0 to 1.6. When a segment has a NACH value that ranges from 1.3 to 1.4 it is typically considered a local high street, while a segment with a NACH value from 1.4 to 1.5 represents a busy street [26]. The values represent how many times each spatial area is used to connect to all other spatial areas. In other words, activity distribution is modeled across the whole spatial structure. Where streets with high values tend to have more movement and pedestrian activities [27]. Red represents routes that are more integrated in the network and are most likely to have high urban activity. Ranging down to the color blue which represents route that have relatively less urban activity. These measures can assist planners in identifying optimum routes of transit in Mansoura city to go along with corridors of high urban activity. This map can also be used to easily communicate planning decisions to local authorities and officials whom have executive authority through offering visual analytical evidence for planning decisions.

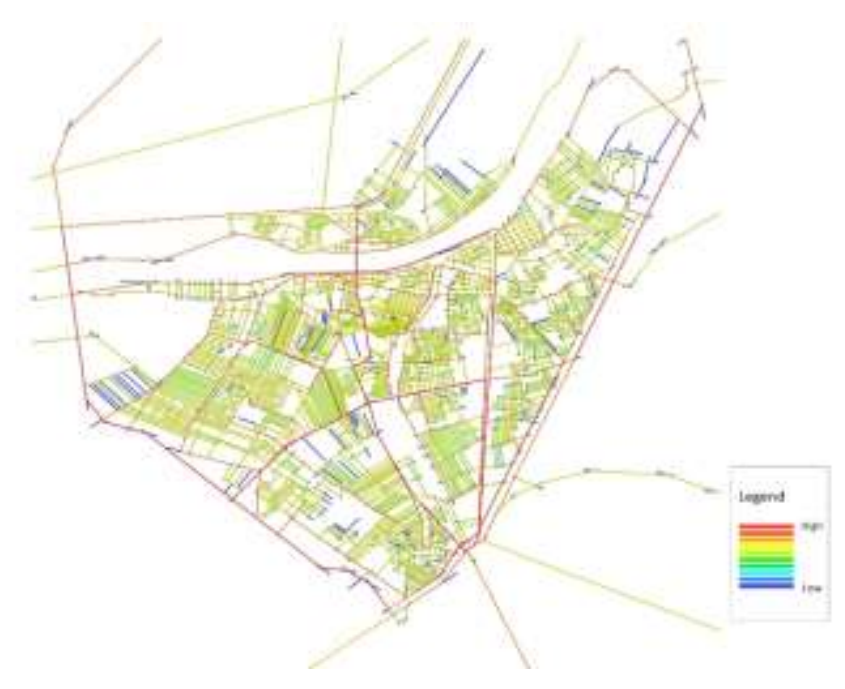

Fig. 6. Normalized Choice analysis at global scale where $r=n$. Source: The Authors, using Depthmap software. Date: April 2020

\section{B. Centrality Index and Optimized Stations}

The index of centrality examines the links inside the spatial network in order to detect spaces which are more accessible to reach from other areas. This measure is calculated by running a segment map analysis using angular integration measure when number of steps was set at 5, which means 5 angular turns from each segment. This number of steps/angular turns focused on the centers on the local scale. Spaces which have high volume of activity and act as gathering points have higher centrality index. Areas with high centrality index has good potential for locating transit stations (see fig.7). Areas with high centrality values were highlighted on the map to indicate areas of proposed transit stations. Centers can be categorized into main centers and sub-centers depending on the resulted measures. This can be utilized in identifying optimum locations for main multi-mode transit stations and smaller transit stops.

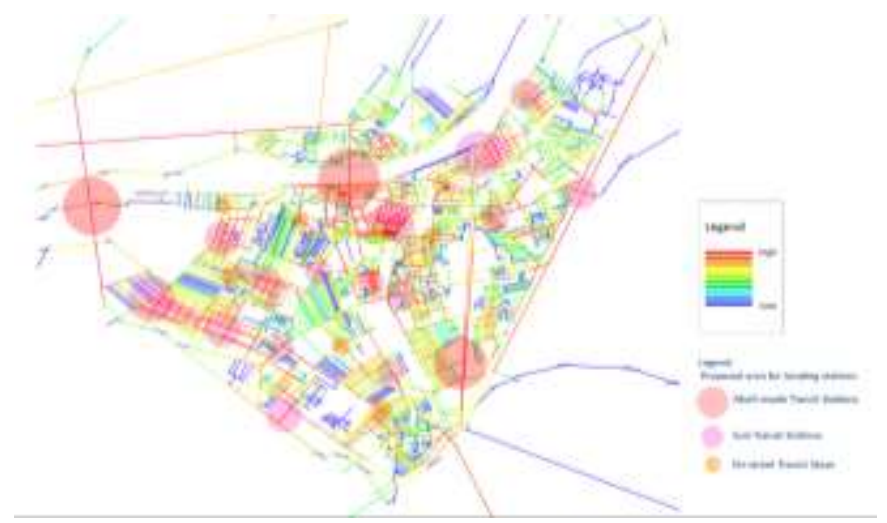

Fig. 7. Integration measure analysis at local scale, using angular step measure where r=5 steps. Source: The Authors, using Depthmap software. April 2020

In order to identify the exact location of the transit stops, further detailed research is needed. Such as land use, space availability, width of pavements, width of the street itself, feasibility, and many other factors need to be taken into 
consideration while locating the exact site of the transit stop. The main benefit of Space Syntax spatial analysis is to identify areas that are more likely to be centers of activity, such as the red circles represent in fig. (7), thereby narrowing down the possible locations for transit stops. It is an added layer of analysis that provides scientific evidence to planners in order to make better informed decisions, however it is not the whole process.

\section{Validation of Analysis Results}

The validation process was achieved through overlaying the analysis results with the manual map made based on the field observations. A scatterplot was generated using both the analysis and observations values in order to test the correlation between them and confirm the validity of the analysis results. The results denote a strong positive correlation where the correlation coefficient $\mathrm{r}=0.7052$ and the squared correlation value $\mathrm{r} 2=0.4973$. In order for correlation to be significantly acceptable values must range from $\mathrm{r}=0.6$ to 0.8 [28], which is achieved in this case. Therefore, results of spatial analysis conducted by Space Syntax can be reliable in making informed decision related to planning transit networks.

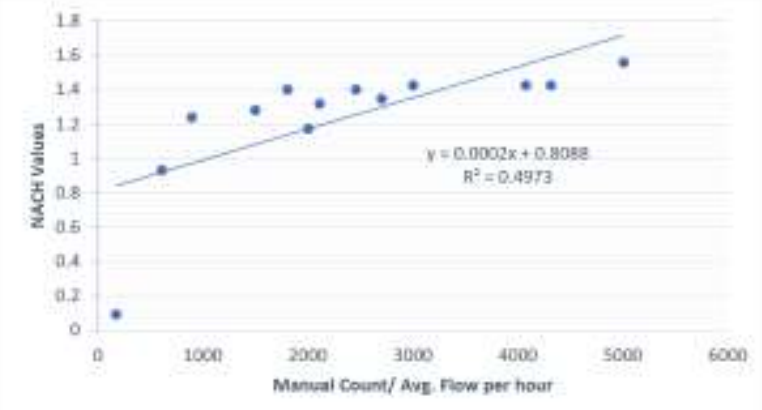

Fig. 8. Scatterplot of NACH values with observed vehicular flow. Source: The Authors using Microsoft Excel software.

\section{DISCUSSION}

\section{A. Potentials}

Space Syntax has shown to offer great potential to be utilized in transportation network planning and assist in the process of identifying optimum transit routes and stations locations. Its potential is based on the fact that Space Syntax can identify relationships between different parts of the network on different scales, model vehicular and pedestrian movement patterns, and easily visualize these relations and measures in a way that is easy to read and understand. The results of the study suggest that Space Syntax enables us to have a comprehensive view of the road network, therefore allowing an easy way to map how specific interventions would impact the whole network. On the other hand, conventional approaches of transport planning would require more time and more resources.

Based on this study and many others, it has been proven that there is a strong correlation between the modeled movement flow distribution and the actual flow of people in real life. In addition, spatial Space Syntax analysis acts as a reliable tool to predict and forecast urban activity and centrality of new city masterplans. Therefore, it can be utilized as an added layer of analysis to the street network in order to identify routes of high urban activity and areas of high urban centrality. Building on that analysis planners can narrow down their choices to plan optimum transit routes and stations. Planners can then conduct further detailed study of the identified areas and choose the exact feasible routes and stations. As a result, Space Syntax spatial analysis can assist planners in making more informed decisions based on measurable evidence while planning transit networks prior to execution.

\section{B. Limitations}

Some limitations of Space Syntax analysis are that it mainly focuses on the topological character of the urban network without giving much influence to other street features, such as obstacles, slops or topological conditions, affecting transportation performance. Other than that Space Syntax configurational analysis does not take into consideration geometrical dimensions (i.e. length, width, number of lanes). It has been established that Space Syntax topological analysis is not an enough tool to conduct an accurate transportation analysis, the reason for which UCL has exported tremendous effort to fix this point. They developed a topo-geometric analysis in Depthmap software, in order to adapt to the kind of analysis needed in transportation and network analysis [23].

Topo-geometric analysis uses both the connectivity and spatial geometry to calculate configurational measures that are approximate to movement flow in patterns in spatial network. While the metric distance captures the exact function and formal properties of the network, topo-geometric analysis links the urban fabric, at different scales, into a whole. Topogeometric analysis has enabled more accurate modeling of movement flow patterns and strengthened Space Syntax applicability in transportation network planning and urban planning in general [29].

\section{Comparison between Space Syntax Approach and Traditional Approach}

In order to summarize the main differences between the Space Syntax approach and the traditionally followed approach, fig. (9) Illustrates the differences in each step (data collection, analysis, results, and evaluation) of the transportation network planning process between both approaches. Space Syntax approach offers an extra dimension to the traditional approach which the concept and tool of spatial analysis. Space Syntax spatial analysis is a strong and reliable tool that provides evidence-based data to planners to make more informed decisions rather that depending solely on the personal cognition and experience.

The spatial analysis results allows planners to model and forecast movement patterns within a city, therefore assists in the process of locating potential transit routes and stations in existing cities and in future ones. Then, additional field studies are needed to ensure the feasibility of the chosen transit routes and stops. Space Syntax analysis is very important in the evaluation process and its results can be visualized in a clear and easy-to-read way that allows planners to easily communicate plans with decision makers. 


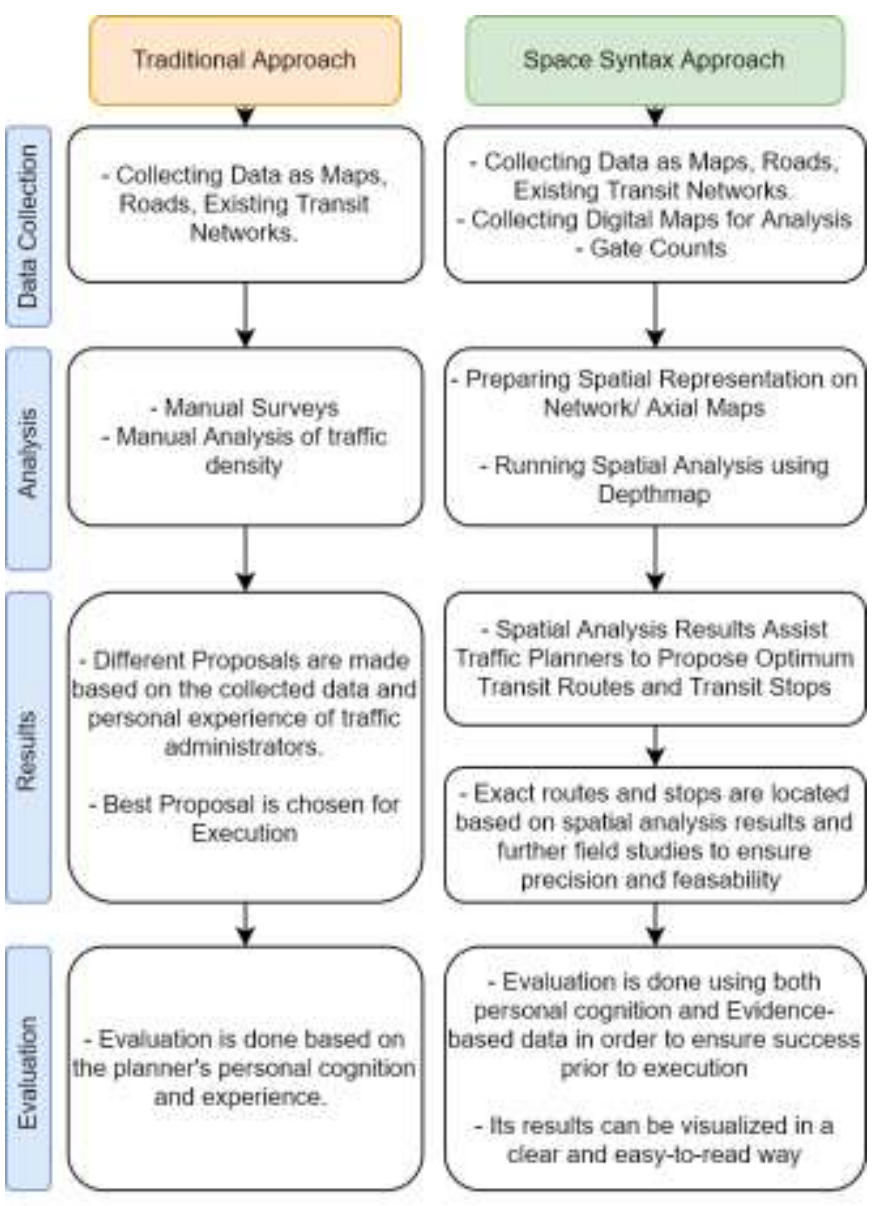

Fig. 9. Diagram Illustrating difference between Space Syntax method and traditional method in planning transit routes and stops.

Source: The Authors.

\section{Conclusion}

This research argues that Space Syntax as a theory, methodology and tool can be used as an evidence-based approach to achieve optimum transportation networks, including optimum routes and transit stations location. The case study was conducted on Mansoura city in April 2020. The spatial configuration analysis conducted by Space Syntax leads to a deeper understanding of the properties of an urban area. As a result of this analysis, numerical values to various spatial indices guide us to more informed decisions when planning a transit network. Two main spatial indices for Mansoura city are measured: urban activity and urban centrality. Spatial analysis was conducted twice, the first time on global scale where $r=n$ to measure urban activity and the second time on local scale where $\mathrm{r}=5$ to measure urban centrality, where $\mathrm{r}=5$ represents the number of angular turns in the street network. Through measuring the index of urban activity we can identify the routes that have highest urban activity therefore are a better choice for transit routes, while measuring the index of urban centrality enables to identify areas that act as centers and sub-centers to the whole city, therefore presenting as a better choice to locate transit stations.
The approach used in this study can be very beneficial in analytical evaluation and policy formation in matters related to transportation network planning. Space Syntax as an analytical modeling tool is based on enabling planners to form a deeper understanding of the urban city as an arrangement of overlapping and interconnected spatial areas. This unique proposition has widened its uses and applications in mobility and transportation and in urban planning in general. Understanding the spatial features of the city gives us a comprehensive idea on how a city works. Therefore, enabling us to take planning decisions that match well with the already existing city structure. As a result, we can maximize the efficiency of the interventions we make to enhance the quality of life in cities and reach the city's full potential as spatial structures that ensure the inhabitants' well-being.

\section{REFERENCES}

[1] R. Hickman and D. Banister, Transport, Climate Change and the City. Routledge, 2014.

[2] D. Banister, "The sustainable mobility paradigm," Transp. Policy, vol. 15, no. 2, pp. 73-80, Mar. 2008, doi: 10.1016/j.tranpol.2007.10.005.

[3] P. Mees, Transport for Suburbia: Beyond the Automobile Age. Earthscan, 2009.

[4] M. Givoni, E. Beyazit, and Y. Shiftan, "The use of state-of-the-art transport models by policymakers - beauty in simplicity?," Plan. Theory Pract., vol. 17, no. 3, pp. 385-404, Jul. 2016, doi: 10.1080/14649357.2016.1188975.

[5] K. Karimi, "A configurational approach to analytical urban design: 'Space syntax' methodology,” URBAN Des. Int., Dec. 2012, Accessed: March. 23, 2020. [Online].

[6] A. Ceder, Public Transit Planning and Operation: Modeling, Practice and Behavior, Second Edition. CRC Press, 2016.

[7] G. Laporte, J. A. Mesa, F. A. Ortega, and F. Perea, "Planning rapid transit networks," Socioecon. Plann. Sci., vol. 45, no. 3, pp. 95-104, Sep. 2011, doi: 10.1016/j.seps.2011.02.001.

[8] R. Hickman, S. Saxena, D. Banister, and O. Ashiru, "Examining transport futures with scenario analysis and MCA," Transp. Res. Part Policy Pract., vol. 46, no. 3, pp. 560-575, Mar. 2012, doi: 10.1016/j.tra.2011.11.006.

[9] B. Hillier, "Spatial sustainability in cities: organic patterns and sustainable forms," Proceedings of the 7th International Space Syntax Symposium, 2009. http://www.sss7.org/Proceedings_list.html (accessed Mar. 17, 2020).

[10] Y. Lerman and I. Omer, "Urban area types and spatial distribution of pedestrians: Lessons from Tel Aviv," Comput. Environ. Urban Syst., vol. 55, pp. 11-23, Jan. 2016, doi: 10.1016/j.compenvurbsys.2015.09.010.

[11] B. Jiang, "Ranking spaces for predicting human movement in an urban environment," Int. J. Geogr. Inf. Sci., vol. 23, no. 7, pp. 823-837, Jul. 2009, doi: 10.1080/13658810802022822.

[12] B. Hillier and S. Iida, "Network and Psychological Effects in Urban Movement," Proceedings of Spatial Information Theory: International Conference, COSIT 2005. Springer-Verlag, Berlin, Germany, Sep. 2005, pp. 475-490, doi: 10.1007/11556114_30.

[13] B. Hillier, A. Penn, J. Hanson, T. Grajewski, and J. Xu, "NATURAL MOVEMENT - OR, CONFIGURATION AND ATTRACTION IN URBAN PEDESTRIAN MOVEMENT,” 1993, doi: 10.1068/b200029.

[14] B. Hillier, "Space is the Machine: A Configurational Theory of Architecture" (C. U. Press Ed.): Cambridge University Press, 1996.

[15] Y. Lerman, Y. Rofè, and I. Omer, "Using Space Syntax to Model Pedestrian Movement in Urban Transportation Planning: Using Space Syntax in Transportation Planning," Geogr. Anal., vol. 46, no. 4, pp. 392410, Oct. 2014, doi: 10.1111/gean.12063.

[16] R. Noichan and B. Dewancker, "Analysis of Accessibility in an Urban Mass Transit Node: A Case Study in a Bangkok Transit Station," Sustainability, vol. 10, no. 12, p. 4819, Dec. 2018, doi: 10.3390/su10124819.

[17] B. Hillier, "Centrality as a process: accounting for attraction inequalities in deformed grids," URBAN Des. Int., vol. 4, no. 3, pp. 107-127, Sep. 1999, doi: 10.1057/udi.1999.19. 
[18] A. Dhanani, L. Tarkhanyan, and L. Vaughan, "Estimating pedestrian demand for active transport evaluation and planning," Transp. Res. Part Policy Pract., vol. 103, pp. 54-69, Sep. 2017, doi: 10.1016/j.tra.2017.05.020

[19] "Egypt: Governorates, Major Cities \& Towns." https://www.citypopulation.de/en/egypt/cities/ (accessed Mar. 30, 2020).

[20] M. Fouda, "Urban Heritage Sites' Sustainable As an Approach to the Intermediate Cities Development (Applied Study for Mansoura -Egypt)," Fac. Eng. - Mansoura Univ., 2011.

[21] M. Elkaramany, "GOVERNMENT PROVISION AND REGULATION OF BUS SERVICE IN CAIRO,” THE AMERICAN UNIVERSITY IN CAIRO, School of Global Affairs and Public Policy, 2015.

[22] K. Al Sayed, A. Turner, B. Hillier, S. Iida, and A. Penn, "Space Syntax methodology", 4th ed. 2014.

[23] R. H. M. Pereira, F. de Holanda, V. de Medeiros, and A. P. B. G. Barros, "The use of space syntax in urban transport analysis: limits and potentials," Brasília: Institute for Applied Economic Research - IPEA. Sampaio, J. C. R. 2015.

[24] A. Turner, "Depthmap 4 - A Researcher's Handbook," p. 58, 2004.

[25] Y. Lebendiger and Y. Lerman, "Applying space syntax for surface rapid transit planning," Transp. Res. Part Policy Pract., vol. 128, pp. 59-72, Oct. 2019, doi: 10.1016/j.tra.2019.07.016.

[26] B. Hillier, T. Yang, and A. Turner, "Normalising least angle choice in Depthmap and how it opens new perspectives on the global and local analysis of city space," vol. 3, pp. 155-193, Dec. 2012.

[27] A. Acharya, K. Karimi, E. Parham, A. Guven, and G. Uyar, "City planning using integrated urban modeling Jeddah structure plan," Proceedings of Acharya2017CityPU. 2017.

[28] S. S. Syed Mahdzar, S. Saari, and S. Jaberolansar, "Planning Integrated Stop Transit for Pedestrian Using Space Syntax Analysis Case Study: Pasir Gudang City," Conference: 14th International Congress of Asian Planning School Association 2017, Asia: Reshaping Urban \& Rural Development through Planning, Beijing, China. 2017.
[29] B. Hillier, A. Turner, T. Yang, and H.-T. Park, "Metric and TopoGeometric Properties of Urban Street Networks: Some convergences, divergences and new results," J. Space Syntax, vol. 1, no. 2, p. 279, 2010.

\section{Title Arabic:}

تحسين شبكات النقل العامة باستخلام التركيب الفراغي كمنهج قائم

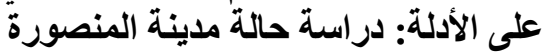

Arabic Abstract:

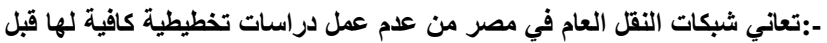

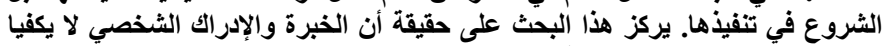

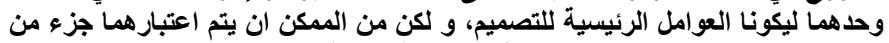

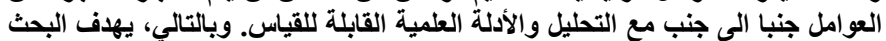

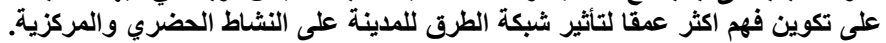

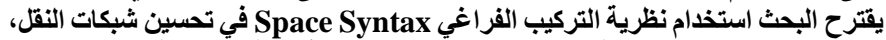

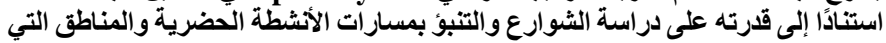

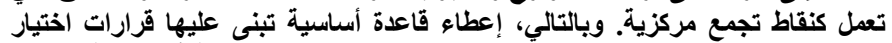

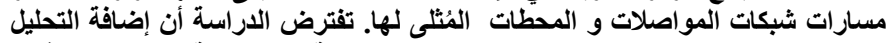

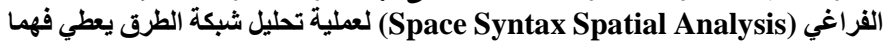

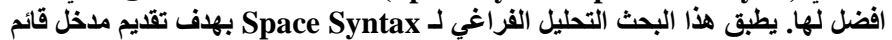

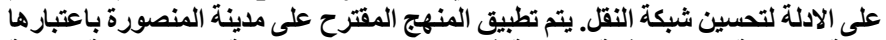

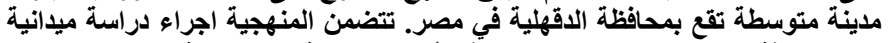

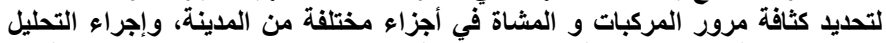

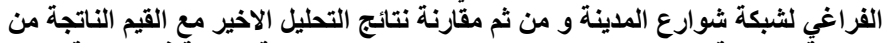

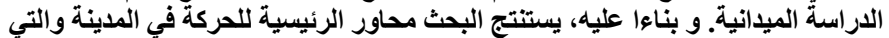

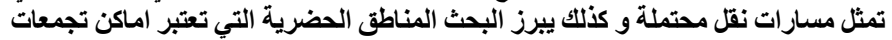

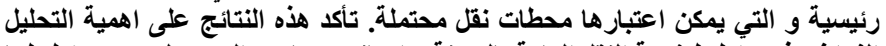

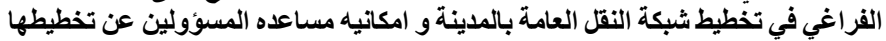

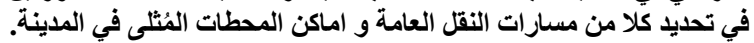

